\title{
Search for Supersymmetry with a compressed mass spectrum in vector boson fusion topology with 1-lepton and 0-lepton final states in pp collisions at $\sqrt{s}=13 \mathrm{TeV}$ with CMS
}

\author{
Priyanka Kumari $^{* \dagger} a$, Nitish Dhingra ${ }^{a, b}$, JB Singh ${ }^{a}$, Vipin Bhatnagar ${ }^{a}$ \\ On behalf of the CMS Collaboration \\ a Panjab University (PU), Chandigarh, India \\ ${ }^{b}$ G.H.G Khalsa College, Ludhiana, India \\ E-mail: priyanka.kumari@cern.ch
}

\begin{abstract}
Searching for Supersymmetry (Susy) is one of the major physics goals of the Large Hadron Collider. A large number of physics analyses are being performed in CMS (and ATLAS) experiments to detect signatures of Susy particles. The search presented here aims for observing Susy particles produced in the Vector Boson Fusion (VBF) topology, leading to a final state having zero or one lepton, large missing $E_{T}$, two jets with high $p_{T}$ and large rapidity separation. The analysis is performed using $35.9 \mathrm{fb}^{-1}$ of proton-proton collision data collected with the CMS detector during year 2016 at center-of-mass energy of $13 \mathrm{TeV}$. The background estimation is performed using data-driven/semi data-driven techniques, and the observed dijet invariant mass as well as lepton transverse mass spectra are found to be consistent with the Standard Model (SM) background predictions and no signal signature is observed. Hence, upper limits are set on the cross sections for chargino $\left(\tilde{\chi}_{1}^{ \pm}\right)$and neutralino $\left(\tilde{\chi}_{2}^{0}\right)$ production along with two associated jets, assuming the lightest scalar leptons to be lighter than $\tilde{\chi}_{1}^{ \pm}$. For a compressed mass spectra in which the mass difference between the Lightest Supersymmetric Particle (LSP) $\tilde{\chi}_{1}^{0}$ and the mass-degenerate particles $\tilde{\chi}_{1}^{ \pm}$and $\tilde{\chi}_{2}^{0}$ is 30 (1) GeV, the most stringent mass upper limit to date is set for the latter two particles.
\end{abstract}

XXIX International Symposium on Lepton Photon Interactions at High Energies - LeptonPhoton2019 August 5-10, 2019

Toronto, Canada

\footnotetext{
* Speaker.

$\dagger$ I am sincerely thankful to CMS Collaboration at LHC experiment, my VBF-SUSY colleagues for the outstanding results presented in this conference, to the LP2019 organizers for a great conference and last but not the least, to the Department of Science and Technology, India for providing me financial support to participate in this major conference in the field of High Energy Physics.
} 


\section{Introduction}

The discovery of the Higgs Boson is considered as the biggest success for the SM as well as for the CMS and ATLAS experiments. Nevertheless, SM still fails to explain a few unsolved problems like unification of forces, hierarchy problem, neutrino oscillations, matter-antimatter asymmetry, nature of dark matter and energy. Supersymmetry (Susy) [1] is considered to be one of the best extensions of the SM that can explain simultaneously the particle nature of dark matter (DM) and solves the gauge hierarchy problem of the SM. Susy associates every SM fermion with its "super-partner" boson and vice-versa which differs by half-integral spin. Despite having attractive features, Susy has no direct evidence of its existence to date. Susy searches at LHC are mostly focused on the colored sector because of its large cross-section, however strongly produced gluinos $(\tilde{g})$, as well as the squarks $(\tilde{q})$ of the first and second generations, have been excluded up to $\sim 2 \mathrm{TeV}$ in certain scenarios. On the other hand, the limits are weaker on the masses of weakly produced charginos $\left(\widetilde{\chi}_{\mathrm{i}}^{ \pm}\right)$and neutralinos $\left(\widetilde{\chi}_{\mathrm{i}}^{0}\right)$. The lightest neutralino $\tilde{\chi}_{1}^{0}$ (LSP) is the canonical DM candidate in R-parity conserving Susy extensions of the SM. CMS and ATLAS have performed various Susy searches covering most of the parameter space. This paper focuses on the result based on the search for the electroweak production of Susy particles in the Vector Boson fusion (VBF) topology using 2016 proton-proton collision data collected with the CMS detector and corresponding to an integrated luminosity of $35.9 \mathrm{fb}^{-1}$ at a center-of-mass energy $\sqrt{s}$ of $13 \mathrm{TeV}$.

\section{Analysis strategy and background estimation}

The charginos and neutralinos are produced in association with two forward jets, in opposite hemispheres of the detector, having large dijet invariant mass and large pseudorapidity. These pair-produced charginos and neutralinos decay to sleptons $(\tilde{l})$ which further decay to the leptons $\left(\tau_{h} / e / \mu\right)$ and lightest neutralinos $\left(\tilde{\chi}_{1}^{0}\right)$ which is LSP. The Susy search with 2016 data at $\sqrt{s}=13$ $\mathrm{TeV}$ has been performed in 4 final states namely: $0 l j j, e j j, \mu j j$, and $\tau_{h} j j$ (hadronically decaying tau). For the Central Selections, events are selected using a trigger based on MET and MHT (magnitude of negative vectorial sum of transverse momentum of all jets) and having a threshold of $120 \mathrm{GeV}$ on both $p_{T, \text { trig }}^{\text {miss }}$ and $H_{T, \text { trig }}^{\text {mis }}$. The compressed mass spectra scenario results in low- $p_{T}$ visible decay products, making it difficult to reconstruct and identify multiple leptons. Hence, events are required to have zero or exactly one well identified soft lepton. In the $\tau_{h} j j$ channel, an additional lepton veto is applied by rejecting events containing a second tau $\left(p_{T}>20 \mathrm{GeV}\right)$, an electron $\left(p_{T}>10 \mathrm{GeV}\right)$, or a muon candidate $\left(p_{T}>8 \mathrm{GeV}\right)$. Similarly, in $e j j$ and $\mu j j$ channels, events are required not to contain another electron, muon, or $\tau_{h}$ candidate. For the $0 l j j$ channel, a well-identified $e, \mu$ or $\tau_{h}$ candidate is rejected. Muon, electron, and $\tau_{h}$ candidates must have $8<p_{T}<40 \mathrm{GeV}, 10<p_{T}<40 \mathrm{GeV}$, and $20<p_{T}<40 \mathrm{GeV}$ and "tight", "medium" and "tight" ID respectively with $|\eta|<2.1$. The events are required to have $E_{T}^{\text {miss }}>250 \mathrm{GeV}$ to suppress the DY $\rightarrow$ $l l$ and QCD multijet backgrounds and no b-jets condition to reduce the $t \bar{t}$ background. A cut on $m_{T}\left(l, E_{T}^{\text {miss }}\right)>110 \mathrm{GeV}$, i.e. beyond the jacobian $m_{W}$ peak (jacobian peak at $p_{T}=m_{w} / 2$ ) to reduce the backgrounds coming from $\mathrm{W}$ boson. The $V B F$ selections require the presence of two VBF jets with $p_{T}>60 \mathrm{GeV}$ and $|\eta|<5.0$ in opposite hemispheres $\left(\eta_{1} \cdot \eta_{2}>0\right)$, large pseudorapidity gap $|\Delta \eta|>3.8$ and separated from the leptons by $\Delta R>0.4$. The VBF dijet candidate with the largest 
dijet mass is chosen and is required to have $m_{j j}>1 \mathrm{TeV}$. The signal region (SR) is defined as the events that satisfy the central and VBF selection criteria. Main backgrounds such as QCD multijet (major for $\tau_{h}$ ), $t \bar{t}$ (large for $e$ and $\mu$ ) and $\mathrm{W}+$ jets are estimated by defining control regions (CR) which are orthogonal to signal region selection. CRs are defined in such a way that they don't bias our $\mathrm{m}_{T}\left(\mathrm{~m}_{j j}\right)$ distribution in the signal region for 1-lepton (0-lepton).

\section{Results and conclusion}

Figure 1 shows the predicted SM background, expected signal, and observed data rates in bins of $m_{T}$ for $\tau_{h}+j j+E_{T}^{\text {miss }}$ final state [2]. The bin sizes in the distributions are chosen to maximize the signal significance of the analysis. No excess of events above the SM prediction in any of the search regions is observed and hence no new physics. For the R-parity conserving MSSM models, results are presented in two scenarios: $(i)$ the "light slepton" model where $\widetilde{\ell}$ is the next-to-lightest Susy particle; and (ii) the "WZ" model where sleptons are too heavy and thus $\widetilde{\chi}_{1}^{ \pm}$and $\widetilde{\chi}_{2}^{0}$ decays proceed via $W^{*}$ and $Z^{*}$. The main difference between the two models is the branching ratio of $\widetilde{\chi}_{1}^{ \pm}$and $\widetilde{\chi}_{2}^{0}$ to leptonic final states. Middel plot in Figure limit1shows that for a compressed mass spectrum scenario, in which $\Delta m=m\left(\tilde{\chi}_{1}^{ \pm}\right)-m\left(\tilde{\chi}_{1}^{0}\right)=1$ (30) $\mathrm{GeV}$ and in which $\tilde{\chi}_{1}^{ \pm}$and $\tilde{\chi}_{2}^{0}$ branching fractions to light sleptons are $100 \%, \tilde{\chi}_{1}^{ \pm}$and $\tilde{\chi}_{2}^{0}$ masses up to 112 (215) $\mathrm{GeV}$ are excluded at $95 \%$ CL. Bottom plot shows that for the scenario where the sleptons are too heavy and decays of the charginos and neutralinos proceed via $W^{*}$ and $Z^{*}$ bosons, $\tilde{\chi}_{1}^{ \pm}$and $\tilde{\chi}_{2}^{0}$ masses up to 112 (175) $\mathrm{GeV}$ are excluded at $95 \% \mathrm{CL}$ for $\Delta m=m\left(\tilde{\chi}_{1}^{ \pm}\right)-m\left(\tilde{\chi}_{1}^{0}\right)=1(30) \mathrm{GeV}$.

\section{References}

[1] Ramond P., "Dual Theory for Free Fermions", Phys. Rev. D, vol. 3, (1971), https://dx.doi.org/10.1103/PhysRevD.3.2415.

[2] The CMS Collaboration, "Search for supersymmetry with a compressed mass spectrum in the vector boson fusion topology with 1-lepton and 0-lepton final states in proton-proton collisions at $\sqrt{s}=13$ TeV”, JHEP, vol. 8 (2019), https://doi.org/10.1007/JHEP08(2019)150. 

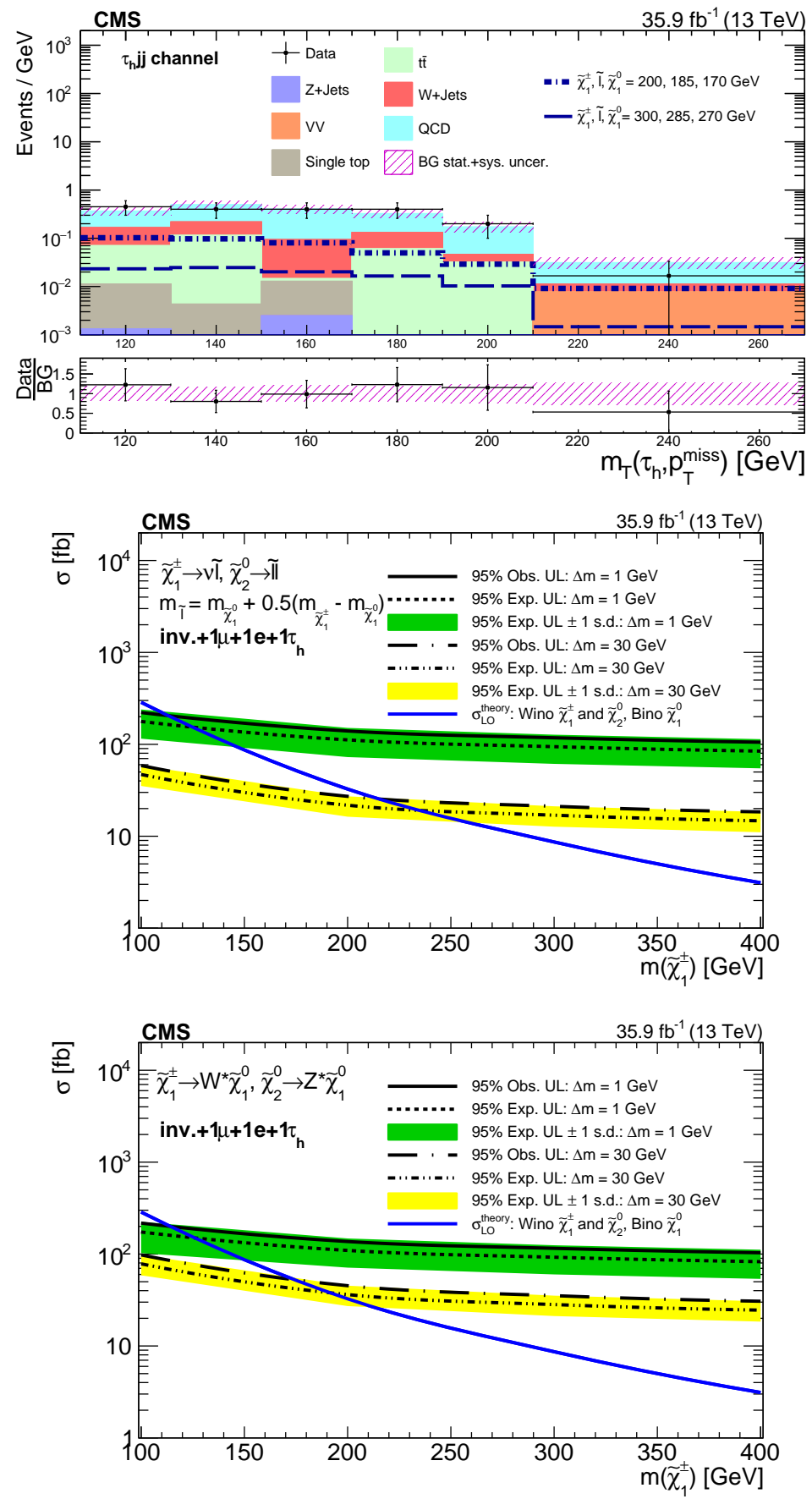

Figure 1: Top plot shows the $m_{T}$ distribution for $\tau_{h}+j j+E_{T}^{\text {miss }}$, middle and bottom shows the limit plots for slepton and WZ model respectively. 